Aceptado: Junio 2018

\title{
La práctica de actividad física y su relación con la satisfacción laboral en una consultora informática
}

\section{The practice of physical activity and its relationship with job satisfaction in a computer consultant}

\author{
Ramón Gómez-Chacón ${ }^{1 *}$, Verónica Morales-Sánchez ${ }^{2}$, Antonio Hernández-Mendo² y Antonio Muñoz-Llerena ${ }^{3}$ \\ 1 CEU Cardenal Spinola. \\ 2 Universidad de Málaga. Facultad de Psicología \\ 3 Agencia para la Gestión, Investigación e Innovación en Servicios Deportivos
}

\begin{abstract}
Resumen: Las organizaciones actuales y modernas están iniciándose en implementar acciones de gestión y promoción de la salud con el objeto de mejorar su calidad de vida y la productividad de la empresa. Una de las variables que impacta en la productividad es la satisfacción laboral, por lo que el propósito de este estudio fue conocer qué tipo de relación existe entre la práctica de actividad física y la satisfacción laboral en Emergya, una consultoría informática. La muestra fueron 152 empleados que contestaron al cuestionario IPAQ para valorar su práctica de actividad física y una pregunta subjetiva sobre la satisfacción laboral. Los resultados mostraron que existen diferencias significativas, siendo los practicantes de actividad física vigorosa $(M=4,19)$, los practicantes de actividad física moderada $(M=$ $4,15)$ y los practicantes de actividad física baja $(M=3,38)$. Por lo tanto los hallazgos concluyen que la práctica de actividad física, de manera vigorosa y moderada influye positivamente en la satisfacción laboral de los empleados. Palabras clave: Actividad física, satisfacción laboral, productividad, orga-
\end{abstract} nización.
Abstract: Current and modern organizations are beginning to implement health management and promotion actions in order to improve their quality of life and the productivity of the company. One of the variables that impacts productivity is job satisfaction, so the purpose of this study was to know what kind of relationship exists between the practice of physical activity and job satisfaction in Emergya, a computer consultancy. The sample consisted of 152 employees who answered the IPAQ questionnaire to assess their physical activity practice and a subjective question about job satisfaction. The results showed that there are significant differences, being the practitioners of vigorous physical activity $(M=4,19)$, the practitioners of moderate physical activity $(M=4,15)$ and the practitioners of low physical activity $(M=3,38)$. Therefore, the findings conclude that the practice of physical activity, in a vigorous and moderate way, positively influences the job satisfaction of employees.

Keywords: Physical activity, job satisfaction, productivity, organization.

\section{Introducción}

Las sociedades actuales y modernas están en continuo proceso de cambio, y con ellas, también las organizaciones. Estos cambios en las organizaciones, a su vez impactan en los puestos de trabajo y por tanto también influyen en la seguridad, la salud y el bienestar de los empleados (Salanova, Martínez y Llorens, 2005). De esta manera, las organizaciones requieren de estructuras y procesos organizacionales flexibles y adaptables, por lo que ya son muchas las empresas que están apostando fuerte por un proceso de cambio organizacional (Arizeta, Portillo y Ayestarán, 2001).

De este modo las empresas españolas llevan iniciándose desde hace ya algunos años en el diseño e implementación de acciones de gestión y promoción de la salud por medio de la actividad física, ya que las organizaciones deben ser además de un lugar de trabajo, un entorno de promoción de la actividad física orientada a una mayor productividad y mejora de la salud, por sus grandes beneficios físicos y psicológicos (Arent,

Dirección para correspondencia [Correspodence address]: Ramón GómezChacón. CEU Cardenal Spínola. E-mail: rgomez@ceuandalucia.es
Landers y Etnier, 2000; Netz, Wu, Becker y Tenenbaum, 2005; Pérez y Devís, 2003).

Concretamente en España se ha impulsado más la implementación de programas de actividad física en las empresas desde la creación de la Estrategia Nutrición, Actividad Física y Prevención de la Obesidad (NAOS). Dicha estrategia desarrolla acciones o intervenciones, en base a la evidencia científica y en todos los ámbitos de la sociedad (familiar, educativo, empresarial, sanitario, laboral, comunitario) que promueven y facilitan opciones a una alimentación variada, equilibrada y a la práctica de actividad fisca, así como a la información más adecuada para ayudar al consumidor a tomar decisiones y elecciones más saludables en entornos que les permitan la adopción de estilos de vida más saludables y activos. Para ello desde la Estrategia NAOS se fomenta la implicación, sinergia y colaboración de todos los sectores y agentes de la sociedad tanto públicos (CCAA, ayuntamientos, otros ministerios) como privados (industria de alimentos y bebidas, sociedades científicas, organizaciones de consumidores, sectores de distribución y restauración, etc.) (Ministerio de Sanidad, Servicios Sociales e Igualdad, 2005). De la misma manera, a nivel 
internacional, en la sesión inaugural de la reunión de la Comisión de Expertos de la Organización Mundial de la Salud (OMS), sobre Promoción de la Salud en el Lugar de Trabajo celebrada en 1987, se reiteró que este organismo consideraba la promoción de la salud de los trabajadores como elemento esencial de los servicios de salud en el trabajo (Organización mundial de la salud, 1988). Del mismo modo, la organización internacional del trabajo (2008), con motivo del XVIII Congreso Mundial sobre Seguridad y Salud en el Trabajo firmaron la Declaración de Seúl sobre Seguridad y Salud en el trabajo,, que afirma específicamente que un ambiente de trabajo seguro y saludable es un derecho humano fundamental.

Por otro lado la satisfacción laboral es un concepto que ha sido estudiado en múltiples ocasiones, en primer lugar por su influencia en el rendimiento laboral, para posteriormente revelarse como una dimensión valiosa en sí misma y como un objetivo de intervención organizacional (Melia y Peiró, 1989).

Locke (1976) define la satisfacción laboral como un estado emocional positivo o placentero resultante de la percepción subjetiva de las experiencias laborales del sujeto o como respuesta afectiva de una persona a su propio trabajo.

Desde un punto de vista llamativo para las organizaciones, es interesante conocer la relación entre la satisfacción laboral y la productividad, de este modo existe una bibliografía extensa que relacionan estas dos variables, del modo que la satisfacción laboral afecta a actitudes y se relaciona positivamente con comportamientos en el trabajo, como el compromiso organizacional (Meyer, Stanley, Herscovitch y Topolnytsky, 2002), el desempeño, el absentismo laboral y la rotación (Harrison, Newman y Roth, 2006; Judge, Thoresen, Bono y Patton, 2001).

Diversos autores discuten sobre el tipo de relación entre satisfacción laboral y la práctica de actividad física. Para Romney y Evans (1996) la satisfacción laboral es importante para el mantenimiento de la salud y la prevención de enfermedades.

Un estudio realizado por Thøgersen-Ntoumani, Fox y Ntoumanis (2005), indicó que el ejercicio físico se relaciona significativamente con altos niveles de autopercepción y con una mayor satisfacción laboral. De la misma manera, un meta-análisis de intervenciones en las organizaciones mostró que la actividad física podría llevar a una menor tensión de trabajo y una mayor satisfacción laboral (Conn, Hafdahl, Cooper, Brown y Lusk, 2009). De Miguel Calvo, Schweiger, De las Mozas y Hernández (2011) sugieren en su estudio que el seguimiento de un programa de ejercicio físico sistematizado y controlado tiene una influencia efectiva sobre la productividad y la satisfacción laboral y el bienestar. Musich, Hook, Baaner y Edington (2006), mostraron que la promoción de la salud en el lugar de trabajo es una medida efectiva para disminuir el absentismo laboral y minimizar la pérdida de productividad. Schultz y Edington (2007), demuestran que programas de bienestar y salud mejoran la satisfacción laboral, que está relacionada con el aumento de la productividad. Por otro lado Thøgersen-Ntoumani, Loughren, Taylor, Duda y Fox (2014) presentaron unos resultados en los cuales sugieren que una intervención de caminar de 16 semanas a la hora del almuerzo en el lugar de trabajo y dirigidos a personas inactivas físicamente les ayudaba a mejorar la percepción del rendimiento de trabajo. Además Gómez-Chacón, GrimaldiPuyana, Bernal-García y Fernández-Gavira (2016) subrayan en su estudio, en una empresa de alimentación, que la práctica de actividad física influye positivamente en la satisfacción laboral.

En base a estas evidencias, donde parece que la práctica de actividad física mejora la satisfacción laboral de los empleados, el objetivo del trabajo es conocer la relación entre la práctica de actividad física y la satisfacción laboral de los empleados de una consultora informática.

\section{Método}

\section{Participantes}

La muestra está compuesta por 152 trabajadores (35 mujeres y 117 hombres), de 250 empleados que tiene la empresa, donde el 42,1\% $(n=64)$ tenía entre 20-29 años; el 42,8\% $(n=65)$ tenía entre 30-39 años; el 11,2\% $(n=17)$ tenía entre 40-49 años, y el 3,9\% $(n=6)$ tenía entre 50-59 años.

\section{Instrumentos}

Como instrumento se utilizó el cuestionario internacional de actividad física versión corta (IPAQ), para evaluar el comportamiento de práctica de actividad física en los trabajadores. El IPAQ tiene una medición aceptable demostrando propiedades para evaluar la percepción subjetiva de los niveles de actividad física (Craig et al., 2003; Brown, Trost, Bauman, Mummery y Owen, 2004). En un estudio de validación se obtuvo una moderada correlación para la actividad física total $(r=0,27 ; p<0,05)$ y tiempo dedicado a actividad física vigorosa $(r=0,38 ; p<0,01)$. Tanto su sensibilidad como su especificidad fueron del 75\% (Román-Viñas, Ribas-Barba, Ngo y Serra-Majem, 2013).

Se les pidió en el cuestionario a los participantes que hicieran un autoinforme sobre la actividad física realizada en la última semana, indicando el número de días y el tiempo en horas y minutos con respecto a episodios de actividad física leve, moderada y alta.

Para conocer el nivel de satisfacción laboral se utilizó una pregunta de elaboración propia y subjetiva, "Indique cuál es su satisfacción laboral" con respuesta escala tipo Likert de siete puntos ( 0 , muy insatisfecho; 6 , muy satisfecho). 


\section{Procedimiento}

Se estableció contacto con la organización para que participará en el estudio en febrero de 2017. Cuando se obtuvo respuesta de aceptación del estudio por parte de la empresa se suministró a los responsables de recursos humanos (RRHH) de la organización el cuestionario vía enlace online, y ellos lo lanzaron a sus empleados dos veces, la primera del 1-5 de febrero y la segunda la semana del 13-19 de febrero. Las respuestas el estudio eran anónimas y se tuvo una tasa de respuesta del 75\%. La recogida de la información fue del 1 de febrero al 20 de febrero de 2017.

\section{Análisis de datos}

Se realizó un análisis de las diferencias significativas mediantes un análisis de la varianza (Anova) entre los diferentes grupos de práctica de actividad física (alta, media y baja) y la satisfacción laboral. Los análisis fueron realizados utilizando el programa estadístico SPSS 24.0.

\section{Resultados}

Los resultados de este estudio muestran que la nacionalidad de los participantes es mayoritariamente española con un $78,9 \%$ (Tabla 1).

Tabla 1. Nacionalidad.

\begin{tabular}{lcc}
\hline & Frecuencia & Porcentaje \\
\hline Española & 120 & 78,9 \\
Norte americana & 3 & 2,0 \\
Asiática & 3 & 2,0 \\
Sudamericana & 23 & 15,1 \\
Otro & 3 & 2,0 \\
Total & 152 & 100,0 \\
\hline
\end{tabular}

El perfil de trabajo estaba dado por técnicos, responsables de área y analistas, destacando los técnicos con un total del 71,7\% (Tabla 2).

Tabla 2. Puesto laboral.

\begin{tabular}{ccc}
\hline & Frecuencia & Porcentaje \\
\hline Analista & 29 & 19,1 \\
Responsable & 14 & 9,2 \\
Técnico/la & 109 & 71,7 \\
Total & 152 & 100,0 \\
\hline
\end{tabular}

El esfuerzo de los empleados al llevar a cabo su labor profesional indica que el $80,9 \%$ no realizan esfuerzos y que su trabajo es sedentario (Tabla 3).

Tabla 3. Esfuerzo laboral.

\begin{tabular}{ccc}
\hline & Frecuencia & Porcentaje \\
\hline Si, mucho & 3 & 2,0 \\
Algo de esfuerzo & 12 & 7,9 \\
Muy poco esfuerzo & 14 & 9,2 \\
No, mi trabajo es sedentario & 123 & 80,9 \\
Total & 152 & 100,0 \\
\hline
\end{tabular}

El salario de los empleados de Emergya muestra que entre la mayoría de los empleados ganan entre 1000-2000 euros con un $91,9 \%$ (Tabla 4).

Tabla 4. Salario.

\begin{tabular}{ccc}
\hline & Frecuencia & Porcentaje \\
\hline MENOS DE 1000 & 35 & 23,0 \\
$1000-1200$ & 24 & 15,8 \\
$1201-1500$ & 42 & 27,6 \\
$1501-2000$ & 39 & 25,7 \\
$2001-2500$ & 9 & 5,9 \\
+3000 & 3 & 2,0 \\
TOTAL & 152 & 100,0 \\
\hline
\end{tabular}

Con respecto a la variable de satisfacción laboral, se muestra que el 38,8\% de los empleados indicaron que estaban algo satisfechos con su empleo, mientras que el $27,6 \%$ se mostraron satisfechos (Tabla 5).

Tabla 5. Satisfacción laboral.

\begin{tabular}{ccc}
\hline & Frecuencia & Porcentaje \\
\hline muy insatisfecho & 3 & 2,0 \\
bastante insatisfecho & 3 & 2,0 \\
algo insatisfecho & 13 & 8,6 \\
insatisfecho & 25 & 16,4 \\
algo satisfecho & 59 & 38,8 \\
satisfecho & 42 & 27,6 \\
muy satisfecho & 7 & 4,6 \\
Total & 152 & 100,0 \\
\hline
\end{tabular}

En la tabla 6 se puede observar la media de los empleados con respecto a la satisfacción laboral. 
Tabla 6. Media satisfacción laboral.

\begin{tabular}{lccccc}
\hline & N & Mínimo & Máximo & Media & $\begin{array}{c}\text { Desviación } \\
\text { estándar }\end{array}$ \\
\hline $\begin{array}{l}\text { Satisfacción } \\
\text { laboral }\end{array}$ & 152 & 0 & 6 & 3,89 & 1,208 \\
\hline
\end{tabular}

De acuerdo con los resultados del IPAQ se observó en los empleados de Emergya que un 34,2\% no realizaban ningún tipo de actividad física, un 44,7\% realizaron actividad física moderada y un 21,1\% realizaban actividad física alta (Tabla 7).

Tabla 7. Tipo de actividad física.

\begin{tabular}{lcc}
\hline & Frecuencia & Porcentaje \\
\hline AF Alta & 32 & 21,1 \\
AF Moderada & 68 & 44,7 \\
AF Baja & 52 & 34,2 \\
Total & 152 & 100,0 \\
\hline
\end{tabular}

Tras el análisis de la varianza ANOVA entre la dimensión satisfacción laboral y la variable práctica de actividad física, se comprobó que existen diferencias significativas entre los tres grupos de práctica de actividad física, mostrando los empleados que practicaron actividad física alta $(M=4,19)$ los índices más altos. A su vez los empleados que practicaron actividad física moderada mostraron índices de $(M=4,15)$, mientras que los empleados que practicaron actividad física baja o no práctica de actividad física mostraron índices bajos $(M=3,28)$ (Tabla 8; Tabla 9).

Tabla 8. Actividad física y satisfacción laboral.

\begin{tabular}{lcc}
\hline & M & D.E \\
AF Alta & 4,19 & 1,306 \\
AF Moderada & 4,15 & 0,885 \\
AF Baja & 3,38 & 1,360 \\
Total & 3,89 & 1,208 \\
\hline
\end{tabular}

Tabla 9. Actividad física y satisfacción laboral.

\begin{tabular}{lccccc}
\hline & $\begin{array}{c}\text { Suma de } \\
\text { cuadrados }\end{array}$ & $g l$ & $\begin{array}{c}\text { Media } \\
\text { cuadrática }\end{array}$ & $F$ & Sig. \\
\hline Entre grupos & 20,604 & 2 & 10,302 & 7,686 & 0,001 \\
Dentro de grupos & 199,712 & 149 & 1,340 & & \\
Total & 220,316 & 151 & & & \\
\hline
\end{tabular}

$\mathrm{p}<, 05, \mathrm{p}<, 01, \mathrm{p}<, 001$

\section{Discusión}

Es un hecho hoy en día que las organizaciones implementen la práctica de actividad física en sus empleados debido a los múltiples beneficios que conllevan, uno de ellos es el impacto que puede producir en la satisfacción de los empleados. Grønningsäter, Hytten, Skauli, CHristensen y Ursin (1992) en un ensayo controlado aleatorio de alta calidad informaron de un efecto negativo significativo de un programa de entrenamiento aeróbico en la satisfacción laboral de los empleados con respecto al grupo control. Por otro lado, dos estudios controlados aleatorios reportados por Rosenfeld, Tenenbaum, Ruskin y Halfon (1990) y Halfon, Rosenfeld, Ruskin y Tenenbaum (1994) mostraron resultados similares a los nuestros, tales que los trabajadores que no participaron en un programa de actividad física en el lugar de trabajo tenían índices de puntuaciones significativamente más bajos que los trabajadores que si lo hacían. Otros estudios, de manera controlada mostraron que no había pruebas concluyentes de efectividad de los programas de actividad física en los lugares de trabajo sobre la satisfacción laboral (Cox, Shephard y Corey, 1981; Cox, Shephard, y Corey, 1987; Shephard, Cox, y Corey, 1981). Los hallazgos principales de este estudio indican que la práctica de actividad física influye positivamente y significativamente en la satisfacción laboral de los empleados, de manera que realizar actividad física, ya sea de manera vigorosa o moderada, se relaciona con empleados con mayores índices de satisfacción laboral, las cuales coinciden con París-Roche, Franco-García y París (2012); Gómez-Chacón, GrimaldiPuyana, Bernal-García y Fernández-Gavira (2016).

\section{Conclusiones}

La conclusión de este estudio indica que la práctica de actividad física, ya sea moderada o alta, influye positivamente en la satisfacción laboral de los empleados, es decir que los empleados que realizan actividad física alta o moderada presentan índices mayores de satisfacción laboral que los que no practican actividad física.

\section{Limitaciones y Futuras líneas de investigación}

Las principales limitaciones de este estudio son la complejidad de acceder y contactar con las empresas, y por otro lado la desconfianza de los responsables de las organizaciones en la toma de datos y los resultados que derivarían de los análisis. Por otro lado la muestra del estudio es pequeńa, al igual que el instrumento para medir la satisfacción laboral utilizando un único indicador y subjetivo, además del instrumento para medir la actividad física que es el IPAQ, que aunque este sobradamente validado y fiable, cuenta con indicadores subjetivos.

Con respecto a las futuras líneas de investigación se plantea utilizar instrumentos más fiables y objetivos para valorar la satisfacción laboral, al igual que usar instrumentos objetivos para valorar la actividad física, como por ejemplo dinamómetros. Por otro lado, estudios que relacionen la práctica 
de actividad física con aspectos psico-sociales. Y por último, respecto a la metodología sería interesante la utilización de diseños Mixed Methods (Morales-Sánchez, Pérez-López, Morquecho-Sánchez y Hernández-Mendo, 2016), novedoso en la gestión deportiva, a través de entrevistas individuales a los empleados, jefes de departamento, gerentes de diferentes empresas, y grupos focales. Utilizando, para ello, herramientas de análisis cualitativo como el ATLAS. ti (Pérez-López, Morales-Sánchez, Anguera y Hernández-Mendo, 2015) y Face Reader en combinación con análisis cuantitativos tipo Análisis de generalizabilidad (Morales-Sánchez, Pérez-López,
Morquecho-Sánchez y Hernández-Mendo, 2016), coordenadas polares (Castellano Paulis y Hernández Mendo, 2003).

\section{Implicaciones para la gestión}

Se recomienda a las organizaciones implementar programas de actividad física para sus empleados ya que mejoraría su estilo vida, consiguiendo empleados más sanos y con una mayor satisfacción laboral y por ende unos mejores trabajadores, repercutiendo en beneficios para la organización.

\section{Referencias}

1. Arent, A. M., Landers, D. M. y Etnier, J. L. (2000). The effects of exercise on mood in olders adults: A meta-analytic review. Journal of Aging and Physical Activity, 8, 407-430.

2. Arizeta, A.G., Portillo, I., y Ayestarán, S. (2001). Cambio organizacional y cultural en un hospital: percepciones y discurso de sus impulsores. Revista de Calidad Asistencial, 16, 22-8.

3. Brown, W.J., Trost, S.G., Bauman, A., Mummery, K. y Owen, N. (2004). Test-retest reliability of four physical activity measures used in population surveys. Journal of Science and Medicine Sports, 7(2), 205-15.

4. Castellano-Paulis, J. y Hernández-Mendo, A. (2003). El análisis de coordenadas polares para la estimación de relaciones en la interacción motriz en fútbol. Psicothema, 15(4), 569-574.

5. Conn, V.S., Hafdahl, A.R., Cooper, P.S., Brown, L.M. y Lusk, S.L. (2009). Meta-Analysis of Workplace Physical Activity Interventions. American Journal of Preventive Medicine, 37, 330-339

6. Cox, M., Shephard, R.J. y Corey, P. (1982). Influence of an employee fitness programme upon fitness, productivity and absenteeism. Ergonomics, 24, 795-806.

7. Cox, M.H., Shephard, R.J. y Corey, P. (1987). Physical activity and alienation in the workplace. The Journal of Sports Medicine and Physical Fitness, 27(4), 429-36.

8. Craig, C.L., Marshall, A.L., Sjostrom, M., Bauman, A.E., Booth, M.L., Ainsworth, B.E., y col. (2003). International physical activity questionnaire: 12-country reliability and validity. Medicine Science Sports Exercise, 35, 1381-95.

9. De Miguel, J. M., Schweiger, I., De las Mozas, O. y Hernández, J. M. (2011). Efecto del ejercicio físico en la productividad laboral y el bienestar. Revista de Psicología del Deporte, 20(2), 589-604.

10. Gómez, R., Grimaldi, M., Bernal, A. y Fernández, J. (2016). La práctica de actividad física y su relación con la satisfacción laboral en una organización de alimentación. Journal of Sports Economics \& Management, 6(2), 85-98.

11. Grønningsäter, H., Hytten, K., Skauli, G., Christensen, C.C. y Ursin, H. (1992). Improved health and coping by physical exercise or cognitive behavioral stress management training in a work environment. Psycholy \& Health, 7, 147-63.

12. Halfon, S.T., Rosenfeld, O., Ruskin, H. y Tenenbaum, G. Daily physical activity program for industrial employees. (1994). En M. Kaneko. (Ed.), Fitness for the aged, disabled, and industrial worker (pp.260-5). Champaign: Human Kinetics.

13. Harrison, D.A., Newman, D.A. y Roth, P.L. (2006). How important are job attitudes? Meta-analytic comparisons of integrative behavioral outcomes and time sequences. Academy of Management Journal, 49(2), $305-325$.

14. Judge, T.A., Thoresen, C.J., Bono, J.E. y Patton, G.K. (2001). The job satisfaction-job performance relationship: A qualitative and quantitative review. Psychological Bulletin, 127(3), 376-407.

15. Locke, E.A. (1976). The nature and causes of job satisfaction. En M. Dunnette M. (Ed.), Handbook of industrial and organizational psycholo$g y$ (pp. 1297-1349). Chicago: Rand McNally.

16. Meliá, J.L. y Peiró, J.M. (1989). El cuestionario de satisfacción S10/12: estructura factorial, fiabilidad y validez. Revista de Psicología del Trabajo $y$ de las Organizaciones, 4(11), 179-187.

17. Meyer, J.P., Stanley, D.J., Herscovitch, L. y Topolnytsky, L. (2002). Affective, continuance, and normative commitment to the organization: A meta-analysis of antecedents, correlates, and consequences. Journal of Vocational Behavior, 61(1), 20-52.

18. Ministerio de Sanidad y Consumo. (2005). Estrategia para la nutrición, actividad fisica y prevención de la obesidad. Recuperado de http://www. aecosan.msssi.gob.es/AECOSAN/web/nutricion/seccion/estrategia_ naos.htm

19. Morales-Sánchez, V., Pérez-López, R., Morquecho-Sánchez, R. y Hernández-Mendo, A. (2016). Generalizabilidad y Gestión Deportiva. Cuadernos de Psicología del Deporte, 16(1), 161-170.

20. Musich, S., Hook, D., Baaner, S. y Edington, D. W. (2006). The association of two productivity measures with health risks and medical conditions in an Australian employee population. American Journal of Health Promotion, 20, 353-363.

21. Netz, Y., Wu, M. J., Becker, B. J. y Tenenbaum, G. (2005). Physical activity and psychological well-being in advanced age: A meta-analysis of intervention studies. Psychology and Aging, 20, 272-284.

22. Organización internacional del trabajo (2008). Declaración de Seúl sobre Seguridad y Salud en el trabajo. Recuperado de http://www.ilo. org/wcmsp5/groups/public/---ed_protect/---protrav/---safework/documents/meetingdocument/wcms_151812.pdf

23. Organización Mundial de la Salud. (1988). Health promotion for working populations: Report of a WHO expert committee. Recuperado de http:// apps.who.int/iris/bitstream/handle/10665/40081/WHO_TRS_765. pdf? sequence $=1 \&$ isAllowed $=y$

24. Paris, F., Franco, A., y París, A. (2012). Valoración socio-económica de un Programa de Actividad Física para los trabajadores de una empresa. Consejo Superior de Deportes.

25. Pérez, V. y Devís, J. (2003). La promoción de la actividad física relacionada con la salud. La perspectiva de proceso y de resultado. Revista Internacional de Medicina y Ciencias de la Actividad Física y del Deporte, 3(10), 69-74.

26. Pérez-López, R., Morales-Sánchez, V, Anguera, M. T. y HernándezMendo, A. (2015). Evaluación de la calidad total en servicios municipales deportivos orientados a la población infantil: Aportaciones desde el análisis cualitativo con ATLAS.ti. Cuadernos de Psicología del Deporte, 15(1), $143-150$. 
27. Román-Viñas, B., Ribas-Barba, L., Ngo, J., y Serra-Majem. (2013). Validación en población catalana del cuestionario internacional de actividad física. Gaceta Sanitaria, 27, 254-257.

28. Romney, D.M., y Evans, D.R. (1996). Toward a general model of health-related quality of life. Quality of Life Research, 5, 235-241.

29. Rosenfeld, O., Tenenbaum, G., Ruskin, H. y Halfon, S.T. (1990). Be havioural modifications following a physical activity programme in the Israeli Pharmaceutical industry. Australian Journal of Science and Medicine in Sport, 22(4), 93-96.

30. Salanova, M., Martínez, I. M. y Llorens, S. (2005). Psicología Organi- zacional Positiva. En F. J. Palací. (Coord.), Psicología de la Organización (pp. 349-376). Madrid: Pearson, Prentice-Hall.

31. Schultz, A.B. y Edington, D.W. (2007). Employee health and presenteeism: a systematic review. Journal of Occupational Rehabilitation, 17, 547-79.

32. Shephard, R.J., Cox, M. y Corey, P. (1982). Fitness program participation: its effect on worker performance. Journal of Occupational Medicine, 23, 359-63.

33. Thøgersen-Ntoumani, C., Fox, K. R. y Ntoumanis, N. (2005). Relationships between exercise and three components of mental well-being in corporate employees. Psychology of Sport and Exercise, 6, 609-627. 